\title{
A finiteness theorem for holomorphic Banach bundles
}

\author{
JÜRGEN LEITERER
}

\begin{abstract}
Let $E$ be a holomorphic Banach bundle over a compact complex manifold, which can be defined by a cocycle of holomorphic transition functions with values of the form id $+K$ where $K$ is compact. Assume that the characteristic fiber of $E$ has the compact approximation property. Let $n$ be the complex dimension of $X$ and $0 \leq q \leq n$. Then: If $V \rightarrow X$ is a holomorphic vector bundle (of finite rank) with $H^{q}(X, V)=0$, then $\operatorname{dim} H^{q}(X, V \otimes E)<\infty$. In particular, if $\operatorname{dim} H^{q}(X, \mathcal{O})=0$, then $\operatorname{dim} H^{q}(X, E)<\infty$.
\end{abstract}

Mathematics Subject Classification (2000): 32F10 (primary); 32C37 (secondary).

\section{Introduction}

By a holomorphic Banach bundle we mean a topological vector bundle $E$ whose characteristic fiber is a complex Banach space $B$ and whose structure is defined by a cocycle of holomorphic transition functions with values in the automorphism group of $B$. Set $\operatorname{rank} E=\operatorname{dim} B$.

Many of the results on holomorphic vector bundles on Stein spaces are valid also for Banach bundles, as it was proved by L. Bungart [1] (1968). This is no more true on non-Stein spaces. For example, it is clear that there is no Kodaira finiteness theorem for holomorphic Banach bundles on compact spaces. Moreover, already on the Riemann sphere $\mathbb{P}^{1}$, there exist holomorphic Banach bundles $E$ with a Hilbert space as characteristic fiber such that $H^{1}\left(\mathbb{P}^{1}, E\right)$ is even not Hausdorff [3]. Hence, to get finiteness theorems for holomorphic Banach bundles, we have to impose additional conditions.

We shall say that a holomorphic Banach bundle with characteristic fiber $B$ is of compact type if it can be defined by a cocycle of holomorphic transition functions with values of the form id $+K$ where $K$ is compact. For such bundles, on the Riemann sphere $\mathbb{P}^{1}$, we have the Gohberg splitting theorem [4] (1964), which is a generalization of the Grothendieck splitting theorem and plays an important role in operator theory:

Received September 11, 2006; accepted in revised form October 30, 2006. 
Gohberg splitting theorem $[4,5,2]$. Any holomorphic Banach bundle of compact type over $\mathbb{P}^{1}$ splits into a finite sum of line bundles and a trivial Banach bundle. ${ }^{1}$

From the Gohberg splitting theorem it follows that, for any holomorphic Banach bundle $E$ of compact type over $\mathbb{P}^{1}$,

$$
\operatorname{dim} H^{q}\left(\mathbb{P}^{1}, E\right) \begin{cases}<\infty & \text { if } q \geq 1 \\ =\infty & \text { if } q=0 \text { and } \operatorname{rank} E=\infty .\end{cases}
$$

So, on the Riemann sphere, for holomorphic Banach bundles of compact type, the Kodaira finiteness theorem is true if $q \geq 1$ and it is not true if $q=0$. The reason is that $H^{q}\left(\mathbb{P}^{1}, \mathcal{O}\right)=0$ if $q \geq 1$ whereas $H^{0}\left(\mathbb{P}^{1}, \mathcal{O}\right) \neq 0$.

Now let $X$ be an arbitrary compact complex manifold. If, for some $q \in \mathbb{N}$, $H^{q}(X, \mathcal{O}) \neq 0$, then it is clear that, for any product bundle of the form $E=X \times B$ where $B$ is an infinite dimensional Banach space,

$$
\operatorname{dim} H^{q}(X, E)=\infty .
$$

Hence there is no Kodaira finiteness theorem also for holomorphic Banach bundle of compact type. However one may expect that the following conjecture ist true:

Conjecture 1.1. If $X$ is a compact complex manifold and $q \in \mathbb{N}$ such that $\operatorname{dim} H^{q}$ $(X, \mathcal{O})=0$, then, for any holomorphic Banach bundle of compact type, $\operatorname{dim} H^{q}$ $(X, E)<\infty$.

In the present paper we prove this conjecture under the additional hypothesis that the characteristic fiber of $E$ has the compact approximation property (see, e.g., $[9]) .^{2}$ Actually we prove the following more general theorem (the conjecture is the special case when $V$ is the trivial line bundle):

Theorem 1.2. Let $X$ be an $n$-dimensional compact complex manifold, $V \rightarrow X a$ holomorphic vector bundle (of finite rank) and $0 \leq q \leq n$ such that

$$
\operatorname{dim} H^{q}(X, V)=0 .
$$

${ }^{1}$ In $[4,5,2]$ this result appears in the language of Riemann-Hilbert factorization. This is equivalent to the formulation given here, because holomorphic Banach bundles over $\mathbb{C}$ are trivial, as it was proved by Bungart [1]. Note also that the first proof of this theorem, given in [4] (1964), uses the additional condition that the bundle can be defined by a cocycle of holomorphic transition functions with values of the form id $+K$ where $K$ can be approximated be finite dimensional operators. (By Enflow's example, found later (1972), there exist Banach spaces such that not any compact operator can be approximated by finite dimensional operators (see, e.g., [9])). This additional condition then was removed in [5].

${ }^{2}$ A Banach space $B$ has the compact approximation property if for each compact set $\Gamma \subseteq B$ and each $\varepsilon>0$, there is a compact endomorphism $K$ of $B$ such that

$$
\|x-K x\| \leq \varepsilon \quad \text { for all } x \in \Gamma .
$$

Note that there exist Banach spaces without approximation property but with compact approximation property (see, e.g., [9]). 
Then, for each holomorphic Banach bundle of compact type $E \rightarrow X$ whose characteristic fiber has the compact approximation property,

$$
\operatorname{dim} H^{q}(X, V \otimes E)<\infty,
$$

and

$$
\operatorname{dim} H^{q}(X, V \otimes E)=0 \quad \text { if } E \text { is trivial. }
$$

Now some remarks about the proof of this theorem. The first ingredient is a general local-global construction of [8]. Under the hypothesis (1.1) this construction gives a global homotopy representation for $V$-valued $(0, q)$-forms. By a general principle (Proposition 2.2), this yields a homotopy representation for $(0, q)$-forms with values in holomorphic Banach bundles of the form $V \otimes E$ where $E$ is a trivial Banach bundle (which completes the proof in this case). The second ingredient is the following observation (Proposition 2.5): let $B$ be a Banach space, $X$ a compact metric space, $\mathcal{C}^{0}(X, B)$ the Banach space of continuous $B$-valued maps on $X$ and $\mathcal{C}^{\alpha}(X, B)$ the Banach space of Hölder- $\alpha$-continuous $B$-valued functions on $X$, $0<\alpha<1$. Further, let $A$ be a bounded linear map from $\mathcal{C}^{0}(X, B)$ to $\mathcal{C}^{\alpha}(X, B)$, and let $K$ be a compact linear endomorphism of $B$. Although then neither $A$ nor the operator of multiplication by $K$ need to be compact as an endomorphism of $\mathcal{C}^{0}(X, B)$, the composition $K A$ is a compact endomorphism of $\mathcal{C}^{0}(X, B)$. It is not clear whether the same is true also for $A K$, but under certain extra conditions, fulfilled in our situation, this is the case (Proposition 2.8).

ACKNOWledgements. I thank Matjaz Erat and Laszlo Lempert for reading the manuscript and many suggestions improving the text.

\section{Preliminaries}

By a Banach space we always mean a complex linear space endowed with a Banach space topology (a norm need not be fixed). The Banach space of bounded linear operators from a Banach space $A$ to a Banach space $B$ will be denoted by $\operatorname{Hom}(A, B)$ and considered as Banach space endowed with the operator norm topology. The subset of isomorphisms of $\operatorname{Hom}(A, B)$ will be denoted by $\operatorname{Iso}(A, B)$ (possibly, it is empty). We set End $(B):=\operatorname{Hom}(B, B)$, and $\operatorname{Aut} B=\operatorname{Iso}(B, B)$.

Let $E$ be a $\mathcal{C}^{\infty}$ Banach bundle over a $\mathcal{C}^{\infty}$ manifold $X$, i.e. a locally trivial bundle whose characteristic fiber is a Banach space $B$ and which can be defined by a cocycle of (Aut $B$ )-valued $\mathcal{C}^{\infty}$ transition functions. If $x \in X$, then the fiber of $E$ over $x$ will be denoted by $E_{x}$. By a fiber norm on $E$ we mean a $\mathcal{C}^{\infty}$ function $\|\cdot\|$ on $E$ such that the restriction to each fiber $E_{x}$ is a norm defining the topology of $E_{x}$.

Let $E, F$ be two $\mathcal{C}^{\infty}$ Banach bundles over a $\mathcal{C}^{\infty}$ manifold $X$. Then we denote by $\operatorname{Hom}(E, F)$ the Banach bundle with $(\operatorname{Hom}(E, F))_{x}=\operatorname{Hom}\left(E_{x}, F_{x}\right), x \in X$. 
The subbundle of isomorphisms in $\operatorname{Hom}(E, F)$ will be denoted by $\operatorname{Iso}(E, F)$. Set End $E=\operatorname{Hom}(E, E)$ and $\operatorname{Aut} E=\operatorname{Iso}(E, E)$.

By a vector bundle $\left(\mathcal{C}^{\infty}\right.$ or holomorphic) we always mean a usual complex vector bundle of finite rank.

Let $E$ be a $\mathcal{C}^{\infty}$ Banach bundle over a $\mathcal{C}^{\infty}$ manifold $X$, and $k \in \mathbb{N} \cup\{\infty\}$ $(\mathbb{N}=\{0,1, \ldots\})$. Then we denote by $\mathcal{C}^{k}(X, E)$ the Fréchet space of global $\mathcal{C}^{k}$ sections of $E$, and, if $k<\infty$ and $0<\alpha<1$, then we denote by $\mathcal{C}^{k+\alpha}(X, E)$ the Fréchet space of global $\mathcal{C}^{k}$ sections of $E$ whose derivatives of order $k$ are Hölder continuous with exponent $\alpha$.

Let $V$ be a $\mathcal{C}^{\infty}$ vector bundle over a $\mathcal{C}^{\infty}$ manifold $X$ and $B$ a Banach space. The fiberwise defined tensor product $V \otimes(X \times B)$ will be simply denoted by $V \otimes B$. For $\psi \in \mathcal{C}^{k}(X, V)$ and $b \in B$, then we have the element $\psi \otimes b$ of the tensor product $\mathcal{C}^{k}(X, V) \otimes B$ of the spaces $\mathcal{C}^{k}(X, V)$ and $B$. On the other hand, we have the fiberwise defined tensor product $\psi \otimes(X \times b) \in \mathcal{C}^{k}(X, V \otimes B)$ of $\psi$ and the constant section $X \times b$ of $X \times B$. It is easy to see that the linear map

$$
\mathcal{C}^{k}(X, V) \otimes B \longrightarrow \mathcal{C}^{k}(X, V \otimes B)
$$

defined by

$$
\psi \otimes b \longrightarrow \psi \otimes(X \times b), \quad \psi \in \mathcal{C}^{k}(X, V), \quad b \in B
$$

is injective, for each $k \in \mathbb{N} \cup\{\infty\}$. Therefore the subspace of $\mathcal{C}^{k}(X, V \otimes B)$ which consists of the sums of the form

$$
\sum_{j=1}^{N} \psi_{j} \otimes\left(X \times b_{j}\right), \quad \psi_{j} \in \mathcal{C}^{k}(X, V), \quad b_{j} \in B,
$$

can be identified with the tensor product $\mathcal{C}^{k}(X, V) \otimes B$. We will do this, and for the now identified products $\psi \otimes b$ and $\psi \otimes(X \times b)$ we will use the simpler notation $\psi \otimes b$. Clearly, for $\operatorname{dim} B=\infty, \mathcal{C}^{k}(X, V) \otimes B$ is strictly smaller than $\mathcal{C}^{k}(X, V \otimes B)$, but we have

Proposition 2.1. Let $V$ be a $\mathcal{C}^{\infty}$ vector bundle over a $\mathcal{C}^{\infty}$ manifold $X, B$ a Banach space and $k \in \mathbb{N} \cup\{\infty\}$. Then the space $\mathcal{C}^{\infty}(X, V) \otimes B$ is dense in $\mathcal{C}^{k}(X, V \otimes B)$ with respect to the $\mathcal{C}^{k}$ topology.

Proof. It is sufficient (by partition of unity) to prove the following local result:

Lemma. Let $U \subseteq \mathbb{R}^{n}$ be an open set and $\mathcal{D}^{k}(U, B)$ the space of $B$-valued $\mathcal{C}^{k}$ maps with compact support in $U, k \in \mathbb{N} \cup\{\infty\}$. Denote by $\mathcal{D}^{k}(U, \mathbb{C}) \otimes B$ the subspace of $\mathcal{D}^{k}(U, B)$ which consists of the finite sums of the form $\sum \psi_{j} b_{j}$ where $\psi_{j} \in \mathcal{D}^{k}(U, \mathbb{C})$ and $b_{j} \in B$. Then $\mathcal{D}^{\infty}(U, \mathbb{C}) \otimes B$ is dense in $\mathcal{D}^{k}(U, B)$ with respect to the $\mathcal{C}^{k}$ topology.

To prove the lemma we fix some norm $\|\cdot\|_{B}$ on $B$ defining the topology of $B$. 
First consider the case $k=0$. Let $f \in \mathcal{D}^{0}(U, B)$ and $\varepsilon>0$ be given. Since $f$ is continuous and has compact support in $U$, we can find a finite open covering $U_{1}, \ldots, U_{N}$ of $U$ such that $\|f(x)-f(y)\| \leq \varepsilon$ whenever $x$ and $y$ belong to the same $U_{j}$. Fix some point $u_{j}$ in each $U_{j}$ such that $f\left(u_{j}\right)=0$ if $U_{j} \cap \operatorname{supp} f=\emptyset$. Take a continuous partition of unity $\left\{\chi_{j}\right\}$ subordinated to the covering $\left\{U_{j}\right\}$ and set

$$
\tilde{f}(x):=\sum_{j=0}^{N} \chi_{j}(x) f\left(u_{j}\right) \quad \text { for } x \in U .
$$

Then it is clear that $\tilde{f} \in \mathcal{D}^{0}(U, \mathbb{C}) \otimes B$ and $\|\tilde{f}(x)-f(x)\|_{B} \leq \varepsilon$ for all $x \in U$.

To prove the lemma for $1 \leq k \leq \infty$, it is sufficiet to do this for $1 \leq k<\infty$. Let such $k$ be given. Let $d \lambda$ be the Euclidean volume form on $\mathbb{R}^{n}$. Fix a nonnegative $\mathcal{C}^{\infty}$-function $\varphi$ on $\mathbb{R}^{n}$ with $\int_{\mathbb{R}^{n}} \varphi d \lambda=1$ and $\varphi(x)=0$ if $|x| \geq 1$. Set $\varphi_{\varepsilon}(x)=\varepsilon^{-n} \varphi(x / \varepsilon)$ for $x \in \mathbb{R}^{n}$ and $\varepsilon>0$. If $u: \mathbb{R}^{n} \rightarrow B$ is continuous and $\varepsilon>0$, then we define

$$
\left(T_{\varepsilon} u\right)(x)=\int_{\mathbb{R}^{n}} \varphi_{\varepsilon}(x-y) u(y) d \lambda(y)=\int_{\mathbb{R}^{n}} \varphi_{\varepsilon}(y) u(x-y) d \lambda(y) .
$$

Now let $f \in \mathcal{D}^{k}(U, B)$ and $\delta>0$ be given. Take $\varepsilon_{0}>0$ so small that the distance between $\operatorname{supp} f$ and the boundary of $U$ is bigger than $2 \varepsilon_{0}$, and let $U^{\prime}$ be the open $\varepsilon_{0}$-neighborhood of $\operatorname{supp} f$. Then, for $\varepsilon<\varepsilon_{0}, T_{\varepsilon} f$ belongs to $\mathcal{D}^{\infty}\left(U^{\prime}, B\right)$. (To see this one has to differentiate under the sign of integration in the first integral of (2.2).) Moreover, for $\varepsilon \rightarrow 0, T_{\varepsilon} f$ tends to $f$ in the $\mathcal{C}^{k}$ topology. (To see this one has to differentiate under the sign of integration in the second integral of (2.2).) Therefore, we can find $0<\varepsilon<\varepsilon_{0}$ with

$$
\left\|f-T_{\varepsilon} f\right\|_{k}<\frac{\delta}{2}
$$

where $\|\cdot\|_{k}$ denotes the $\mathcal{C}^{k}$-norm with respect to $\|\cdot\|_{B}$. As the lemma is already proved for $k=0$, moreover we can find a sequence $g_{j} \in \mathcal{D}^{0}\left(U^{\prime}, \mathbb{C}\right) \otimes B$ such that

$$
\lim _{j \rightarrow \infty} \max _{x \in U^{\prime}}\left\|f(x)-g_{j}(x)\right\|_{B}=0 .
$$

Set $f_{j}=T_{\varepsilon} g_{j}$. Then $f_{j} \in \mathcal{D}^{\infty}(U, \mathbb{C}) \otimes B$ for all $j$, and it follows from (2.3)

$$
\left\|f-f_{j}\right\|_{k}<\left\|T_{\varepsilon} f-f_{j}\right\|_{k}+\frac{\delta}{2}=\left\|T_{\varepsilon}\left(f-g_{j}\right)\right\|_{k}+\frac{\delta}{2} .
$$

By differentiation under the sign of integration in the first integral of (2.2) and by (2.4) it follows that

$$
\lim _{j \rightarrow \infty}\left\|T_{\varepsilon}\left(f-g_{j}\right)\right\|_{k}=0 .
$$

Hence, we can choose $j$ so large that $\left\|f-f_{j}\right\|_{k}<\delta$. 
Proposition 2.2. Let $V$ and $V^{\prime}$ be $\mathcal{C}^{\infty}$ vector bundles over $\mathcal{C}^{\infty}$ manifolds $X$ and $X^{\prime}$ respectively. Let $k, k^{\prime} \in \mathbb{N}, 0 \leq \alpha<1$, and let

$$
A: \mathcal{C}^{k}(X, V) \longrightarrow \mathcal{C}^{k^{\prime}+\alpha}\left(X^{\prime}, V^{\prime}\right)
$$

be a continuous linear operator. Then, for each Banach space B, there exists a uniquely determined continuous linear operator

$$
A_{B}: \mathcal{C}^{k}(X, V \otimes B) \longrightarrow \mathcal{C}^{k^{\prime}+\alpha}\left(X^{\prime}, V^{\prime} \otimes B\right)
$$

such that

$$
A_{B}=A \otimes \text { id } \quad \text { on } \quad \mathcal{C}^{k}(X, V) \otimes B
$$

where

$$
A \otimes \mathrm{id}: \mathcal{C}^{k}(X, V) \otimes B \longrightarrow \mathcal{C}^{k+\alpha}\left(X^{\prime}, V^{\prime}\right) \otimes B
$$

is the algebraic tensor product of $A$ and the identical endomorphism of $B$.

Proof. Since, by Proposition $2.1, \mathcal{C}^{k}(X, V) \otimes B$ is dense in $\mathcal{C}^{k}(X, V \otimes B)$, it is sufficient to prove that $A \otimes$ id is continuous with respect to the $\mathcal{C}^{k}$ topology in the source space and the $\mathcal{C}^{k^{\prime}+\alpha}$ topology in the target space.

Let $n=\operatorname{dim} X, n^{\prime}=\operatorname{dim} X^{\prime}, r=\operatorname{rank} V, r^{\prime}=\operatorname{rank} V^{\prime}$. Fix some norm $\|\cdot\|$ defining the topology of $B$. Denote by $B^{*}$ the space of continuous linear functionals $\Phi: B \rightarrow \mathbb{C}$ endowed with the norm $\|\Phi\|:=\sup _{x \in B,\|x\|=1}|\Phi(x)|$.

Let $l, m \in \mathbb{N}, \omega \subset \subset \mathbb{R}^{m}$ a compact set and $f$ a $B$-valued $\mathcal{C}^{l}$ map in a neighborhood of $\omega$. Then we set (using the usual notations for partial derivatives)

$$
\rho_{\omega, l}(f)=\max _{0 \leq|\beta| \leq l} \max _{\zeta \in \omega}\left\|D^{\beta} f(\zeta)\right\|
$$

and, if $\alpha>0$ and $f$ is even of class $\mathcal{C}^{l+\alpha}$,

$$
\rho_{\omega, l+\alpha}(f)=\max \left(\rho_{\omega, l}(f), \max _{|\beta|=l} \sup _{\zeta, \eta \in \omega, \zeta \neq \eta} \frac{\left\|D^{\beta} f(\zeta)-D^{\beta} f(\eta)\right\|}{|\zeta-\eta|^{\alpha}}\right) .
$$

In the same way we define $\rho_{\omega, l}(f)$ and $\rho_{\omega, l+\alpha}(f)$ if $f$ is a scalar function, using the modulus $|\cdot|$ of complex numbers instead of the norm $\|\cdot\|$. By the Hahn-Banach theorem,

$$
\left\|D^{\beta} f(\zeta)\right\|=\max _{\Phi \in B^{*},\|\Phi\|=1}\left|\Phi\left(D^{\beta} f(\zeta)\right)\right|=\max _{\Phi \in B^{*},\|\Phi\|=1}\left|D^{\beta}(\Phi \circ f)(\zeta)\right|
$$

for all $\zeta \in \omega$ and all multi-indices $\beta$ with $0 \leq|\beta| \leq l$. Hence

$$
\rho_{\omega, l}(f)=\max _{\Phi \in B^{*},\|\Phi\|=1} \rho_{\omega, l}(\Phi \circ f) .
$$


If $\alpha>0$ and $f$ is even of class $\mathcal{C}^{l+\alpha}$, then moreover

$$
\begin{aligned}
\sup _{\zeta, \eta \in \omega, \zeta \neq \eta} & \frac{\left\|D^{\beta} f(\zeta)-D^{\beta} f(\eta)\right\|}{|\zeta-\eta|^{\alpha}} \\
& =\sup _{\zeta, \eta \in \omega, \zeta \neq \eta} \max _{\Phi \in B^{*},\|\Phi\|=1} \frac{\left\|D^{\beta}(\Phi \circ f)(\zeta)-D^{\beta}(\Phi \circ f)(\eta)\right\|}{|\zeta-\eta|^{\alpha}} \\
& =\sup _{\Phi \in B^{*},\|\Phi\|=1} \sup _{\zeta, \eta \in \omega, \zeta \neq \eta} \frac{\left\|D^{\beta}(\Phi \circ f)(\zeta)-D^{\beta}(\Phi \circ f)(\eta)\right\|}{|\zeta-\eta|^{\alpha}}
\end{aligned}
$$

and therefore

$$
\rho_{\omega, l+\alpha}(f)=\sup _{\Phi \in B^{*},\|\Phi\|=1} \rho_{\omega, l+\alpha}(\Phi \circ f) .
$$

Fix a family $\left\{\left(U_{j}, \omega_{j}, \varphi_{j}, e_{j}\right)\right\}_{j \in I}$ such that

- $\left\{U_{j}\right\}_{j \in I}$ is a locally finite open covering of $X$;

- each $\omega_{j}$ is a compact subset of $U_{j}$, and $X=\bigcup_{j \in I} \omega_{j}$;

- each $\varphi_{j}$ is a system $\varphi_{j}=\left(\varphi_{j 1}, \ldots, \varphi_{j n}\right)$ of real $\mathcal{C}^{\infty}$ coordinates on $U_{j}$;

- each $e_{j}$ is a $\mathcal{C}^{\infty}$ frame $e_{j}=\left(e_{j 1}, \ldots, e_{j r}\right)$ of $V$ over $U_{j}(r$ is the rank of $V)$.

For $X^{\prime}$ and $V^{\prime}$ we fix a corresponding family $\left\{\left(U_{j}^{\prime}, \omega_{j}^{\prime}, \varphi_{j}^{\prime}, e_{j}^{\prime}\right)\right\}_{j \in I^{\prime}}$.

If $f$ is a section of $V$ over some $\Omega \subseteq X$, then, over each $U_{j} \cap \Omega$, it can be written in the form $f=\sum_{v=1}^{r} f_{j v} e_{j v}$ where $f_{j 1}, \ldots, f_{j r}$ are uniquely determined scalar functions on $U_{j} \cap \Omega$. These functions $f_{j v}$, will be called the coefficients of $f$. Similarly, if $f$ is a section of $V \otimes B$ over some $\Omega \subseteq X$, then by the coefficients of $f$ we mean the uniquely determined maps $f_{j v}: U_{j} \cap \Omega \rightarrow B$ with $f=\sum_{v=1}^{r} e_{j v} \otimes$ $f_{j v}$. If $f$ belongs to one of the spaces $\mathcal{C}^{k}(X, V)$ or $\mathcal{C}^{k}(X, V \otimes B)$, then we define

$$
\rho_{j}(f)=\max _{\nu=1, \ldots, r} \rho_{\omega_{j}, k}\left(f_{j v} \circ \varphi_{j}^{-1}\right) \quad \text { for all } j \in I
$$

where $f_{j v}$ are the coefficients of $f$. The so defined family of semi norms $\left\{\rho_{j}\right\}_{j \in I}$ defines the topologies of $\mathcal{C}^{k}(X, V)$ and $\mathcal{C}^{k}(X, V \otimes B)$.

Similarly, using the family $\left\{\left(U_{j}^{\prime}, \omega_{j}^{\prime}, \varphi_{j}^{\prime}, e_{j}^{\prime}\right)\right\}_{j \in I^{\prime}}$, we define a family of semi norms $\left\{\rho_{j}^{\prime}\right\}_{j \in I^{\prime}}$ defining the topologies of $\mathcal{C}^{k^{\prime}+\alpha}\left(X^{\prime}, V^{\prime}\right)$ and $\mathcal{C}^{k^{\prime}+\alpha}\left(X^{\prime}, V^{\prime} \otimes B\right)$.

Let $\Phi \in B^{*}$. Then we denote by $\Phi_{V}$ the global section of $\operatorname{Hom}(V \otimes B, V)$ which is the fiberwise tensor product of the identity section of End $V$ and $\Phi$, i.e. if a section $f$ of $V \otimes B$ is written as a finite sum $f=\sum_{\mu} \psi_{\mu} \otimes b_{\mu}$ where the $\psi_{\mu}$ are sections of $V$ and the $b_{\mu}$ are $B$-valued maps, then

$$
\Phi_{V} f=\sum_{\mu}\left(\Phi \circ b_{\mu}\right) \psi_{\mu} .
$$

In the same way, we define a global section $\Phi_{V^{\prime}}$ of $\operatorname{Hom}\left(V^{\prime} \otimes B, V^{\prime}\right)$. 
Let $f \in \mathcal{C}^{k}(X, V \otimes B)$ and let $f_{j v}$ be the coefficients of $f$. Then, by (2.8), $\Phi \circ f_{j v}$ are the coefficients of $\Phi_{V} f$. By (2.6) this implies, for all $j \in I$,

$$
\rho_{j}(f)=\max _{\nu=1, \ldots, r} \sup _{\Phi \in B^{*},\|\Phi\|=1} \rho_{\omega_{j}, k}\left(\Phi \circ f_{j \nu} \circ \varphi_{j}^{-1}\right)=\sup _{\Phi \in B^{*},\|\Phi\|=1} \rho_{j}\left(\Phi_{V} f\right) .
$$

Similarly, if $f \in \mathcal{C}^{k^{\prime}+\alpha}\left(X^{\prime}, V^{\prime} \otimes B\right)$ and $j \in I^{\prime}$, then, by (2.8) and (2.7),

$$
\rho_{j}^{\prime}(f)=\sup _{\Phi \in B^{*},\|\Phi\|=1} \rho_{j}^{\prime}\left(\Phi_{V^{\prime}} f\right) .
$$

Let $f \in \mathcal{C}^{k}(X, V) \otimes B$ and $\Phi \in B^{*}$. Then $f$ can be written as a finite sum

$$
f=\sum_{\mu} \psi_{\mu} \otimes b_{\mu} \quad \text { with } \psi_{\mu} \in \mathcal{C}^{k}(X, V) \text { and } b_{\mu} \in B,
$$

and, by (2.8) and the corresponding relation for $\Phi_{V^{\prime}}$, it follows that

$$
\Phi_{V} f=\sum_{\mu}\left(\Phi b_{\mu}\right) \psi_{\mu} \quad \text { and } \quad \Phi_{V^{\prime}}(A \otimes \mathrm{id}) f=\sum_{\mu}\left(\Phi b_{\mu}\right)\left(A \psi_{\mu}\right)
$$

Since the $\Phi b_{\mu}$ are numbers and $A$ is linear, this implies that

$$
\Phi_{V^{\prime}}(A \otimes \mathrm{id}) f=A\left(\sum_{\mu}\left(\Phi b_{\mu}\right) \psi_{\mu}\right)=A \Phi_{V} f .
$$

Now, to prove the continuity of $A \otimes \mathrm{id}$, consider an arbitrary $\kappa \in I^{\prime}$. We have to find a finite number of indices $j_{1}, \ldots, j_{N} \in I$ and a constant $C<\infty$ such that

$$
\rho_{\kappa}^{\prime}((A \otimes \mathrm{id}) f) \leq C \max _{\nu=1, \ldots, N} \rho_{j_{v}}(f)
$$

for all $f \in \mathcal{C}^{k}(X, V) \otimes B$. Since $A$ is continuous, there exist a finite number of indices $j_{1}, \ldots, j_{N} \in I$ and a constant $C<\infty$ such that

$$
\rho_{\kappa}^{\prime}(A v) \leq C \max _{v=1, \ldots, N} \rho_{j_{v}}(v)
$$

for $v \in \mathcal{C}^{k}(X, V)$. Now let $f \in \mathcal{C}^{k}(X, V) \otimes B$. Then, by (2.11) and (2.13),

$$
\rho_{\kappa}^{\prime}\left(\Phi_{V^{\prime}}(A \otimes \mathrm{id}) f\right)=\rho_{\kappa}^{\prime}\left(A \Phi_{V} f\right) \leq C \max _{\nu=1, \ldots, N} \rho_{j_{\nu}}\left(\Phi_{V} f\right) \quad \text { for all } \Phi \in B^{*},
$$

and the required estimate (2.12) now follows by (2.9) and (2.10).

Definition 2.3. Let $E$ and $F$ be $\mathcal{C}^{\infty}$ Banach bundles over a $\mathcal{C}^{\infty}$ manifold $X$. A section $K: X \rightarrow \operatorname{Hom}(E, F)$ will be called fiber compact if, for each $\zeta \in X$, the value $K(\zeta)$ is a compact operator between the fibers $E_{\zeta}$ and $F_{\zeta}$. 
Lemma 2.4. Let $E$ and $E^{\prime}$ be $\mathcal{C}^{\infty}$ Banach bundles over a $\mathcal{C}^{\infty}$ manifold $X$, and let $K: X \rightarrow \operatorname{Hom}\left(E, E^{\prime}\right)$ be a section which is fiber compact and continuous with respect to the operator norm topology. Further, let $\|\cdot\|$ be a fiber norm on E. Then, for each compact set $\omega \subset \subset X$, the set

$$
\left\{K(\zeta) v \mid \zeta \in \omega, v \in E_{\zeta},\|v\| \leq 1\right\}
$$

is precompact in $E^{\prime}$.

Proof. Since $\omega$ is compact, after passing to local trivializations, we may assume that $X$ is an open subset of $\mathbb{R}^{n}$ and the bundles $E$ and $F$ are trivial, i.e. $E=X \times B$ and $E^{\prime}=X \times B^{\prime}$ for some Banach spaces $B$ and $B^{\prime}$, and $K$ can be considered as a continuous map $K: X \rightarrow \operatorname{Hom}\left(B, B^{\prime}\right)$. Assume that some norms are fixed on $B$ and $B^{\prime}$ which we both denote by $\|\cdot\|$. Also the corresponding operator norm on $\operatorname{Hom}\left(B, B^{\prime}\right)$ will be denoted by $\|\cdot\|$. Then we have to prove that the set

$$
\{K(\zeta) v \mid \zeta \in \omega, v \in B,\|v\| \leq 1\}
$$

is precompact in $B^{\prime}$. Let $\varepsilon>0$ be given. As the values of $K$ are compact, for each $\eta \in X$, there is a finite set $\Lambda(\eta) \subseteq B^{\prime}$ such that

$$
\{K(\eta) v \mid v \in B,\|v\| \leq 1\}
$$

is contained in the $\varepsilon / 2$-neighborhood of $\Lambda(\eta)$. Since $K$ is continuous, for each $\eta \in X$, we can find a neighborhood $U(\eta)$ of $\eta$ such that

$$
\|K(\zeta)-K(\eta)\| \leq \frac{\varepsilon}{2} \quad \text { for all } \zeta \in U(\eta) .
$$

Then $\|K(\zeta) v-K(\eta) v\| \leq \varepsilon / 2$ for all $v \in B$ with $\|v\| \leq 1$, and, hence, the set

$$
\{K(\zeta) v \mid \zeta \in U(\eta), v \in B,\|v\| \leq 1\}
$$

is contained in the $\varepsilon$-neighborhood of $\Lambda(\eta)$. Since $\omega$ is compact, there is a finite number of points $\eta_{1}, \ldots, \eta_{N} \in \omega$ such that $\omega$ is covered by $U\left(\eta_{1}\right), \ldots, U\left(\eta_{N}\right)$. Then the set

$$
\bigcup_{j=1}^{N}\left\{K(\zeta) v \mid \zeta \in U\left(\eta_{j}\right), v \in B,\|v\| \leq 1\right\}
$$

is contained in the $\varepsilon$-neighborhood of the finite set $\Lambda\left(\eta_{1}\right) \cup \ldots \cup \Lambda\left(\eta_{N}\right)$. If follows that the set (2.15) is contained in the $\varepsilon$-neighborhood of this finite set.

Proposition 2.5. Let $E, E^{\prime}$, $E^{\prime \prime}$ be $\mathcal{C}^{\infty}$ Banach bundles over a compact $\mathcal{C}^{\infty}$ manifold $X$ and $0<\alpha<1$. Let $K: X \rightarrow \operatorname{Hom}\left(E^{\prime}, E^{\prime \prime}\right)$ be a section which is fiber compact and Hölder continuous with exponent $\alpha$ with respect to the operator norm topology. Further let

$$
A: \mathcal{C}^{0}(X, E) \longrightarrow \mathcal{C}^{\alpha}\left(X, E^{\prime}\right)
$$

be a continuous linear operator. Then $K A$ is compact as an operator between $\mathcal{C}^{0}(X, E)$ and $\mathcal{C}^{0}\left(X, E^{\prime \prime}\right)$. 
Proof. Let a bounded sequence $\left(f_{j}\right)_{j \in \mathbb{N}}$ in $\mathcal{C}^{0}(X, E)$ be given. Then $\left(A f_{j}\right)_{j \in \mathbb{N}}$ is a bounded sequence in $\mathcal{C}^{\alpha}\left(X, E^{\prime}\right)$. In particular, $\left(A f_{j}\right)_{j \in \mathbb{N}}$ is bounded in $\mathcal{C}^{0}\left(X, E^{\prime}\right)$. As $X$ is compact, it follows, by Lemma 2.4 , that

$$
\Lambda:=\left\{\left(K A f_{j}\right)(\zeta) \mid \zeta \in X, \quad j \in \mathbb{N}\right\}
$$

is precompact in $E^{\prime \prime}$. Since $K$ is Hölder continuous with exponent $\alpha$ and $\left(A f_{j}\right)_{j \in \mathbb{N}}$ is a bounded sequence in $\mathcal{C}^{\alpha}\left(X, E^{\prime}\right)$, now we see that $\left(K A f_{j}\right)_{j \in \mathbb{N}}$ is a bounded sequence in $\mathcal{C}^{\alpha}\left(X, E^{\prime \prime}\right)$ all values of which belong to the precompact set $\Lambda$. By Ascoli's theorem this sequence has a uniformly convergent subsequence.

Lemma 2.6. Let $V$ be a $\mathcal{C}^{\infty}$ vector bundle over a $\mathcal{C}^{\infty}$ manifold $X, B$ a Banach space and $F$ a linear subspace of $B$. Let $\|\cdot\|$ be a fiber norm on $V \otimes B, \varepsilon>0$ and $f: X \rightarrow V \otimes B$ a continuous section such that

$$
\inf _{v \in(V \otimes F)_{\zeta}}\|f(\zeta)-v\|<\varepsilon \quad \text { for all } \zeta \in X .
$$

Then there exists a continuous section $f_{\varepsilon}: X \rightarrow V \otimes F$ such that

$$
\left\|f(\zeta)-f_{\varepsilon}(\zeta)\right\|<\varepsilon \quad \text { for all } \zeta \in X
$$

Proof. By hypothesis, for each $\zeta \in X$, we can fix a vector $v_{\zeta} \in(V \otimes F)_{\zeta}$ such that $\left\|f(\zeta)-v_{\zeta}\right\|<\varepsilon$. Further, for each such vector, we can find a continuous section $f_{\zeta}: X \rightarrow V \otimes F$ with $f_{\zeta}(\zeta)=v_{\zeta}$. Then, for each $\zeta \in X$, we choose a neighborhood $U_{\zeta}$ of $\zeta$ so small that $\left\|f(\eta)-f_{\zeta}(\eta)\right\|<\varepsilon$ for all $\eta \in U_{\zeta}$. Finally, take a continuous partition of unity $\left\{\chi_{\zeta}\right\}_{\zeta \in X}$ subordinated to the covering $\left\{U_{\zeta}\right\}_{\zeta \in X}$, and set $f_{\varepsilon}(\eta)=\sum_{\zeta \in X} \chi_{\zeta}(\eta) f_{\zeta}(\eta)$ for $\eta \in X$.

Lemma 2.7. Let $V$ be a $\mathcal{C}^{\infty}$ vector bundle over a compact $\mathcal{C}^{\infty}$ manifold $X, B$ a Banach space, $\|\cdot\|$ a fiber norm on $V \otimes B$, and $\omega$ a compact subset of the total space of the bundle $V \otimes B$. Then, for each $\varepsilon>0$, there exists a finite dimensional subspace $F$ of $B$ such that

$$
\inf _{w \in(V \otimes F)_{\zeta}}\|v-w\|<\varepsilon \quad \text { for all } \zeta \in X \text { and } v \in \omega \cap(V \otimes B)_{\zeta} .
$$

Proof. Let $r$ be the rank of $V$ and $B^{r}$ the direct sum of $r$ copies of $B$. Fix some norm $\|\cdot\|_{B^{r}}$ of $B^{r}$. Take a Riemann metric on $X$ and let $d_{X}$ be the corresponding distance function. Setting

$$
d((\zeta, v),(\eta, w))=d_{X}(\zeta, \eta)+\|v-w\|_{B^{r}} \quad \text { for } \zeta, \eta \in X \text { and } v, w \in B^{r}
$$

we choose a metric $d$ on $X \times B^{r}$. Since $X$ is compact, we can find a finite open covering $\widetilde{U}_{1}, \ldots, \widetilde{U}_{N}$ of $X$ and local $\mathcal{C}^{\infty}$ trivializations $\theta_{j}:\left.V\right|_{\widetilde{U}_{j}} \rightarrow \widetilde{U}_{j} \times \mathbb{C}^{r}$ of $V$. Setting $\Theta_{j}=\theta_{j} \otimes$ id we get trivializations

$$
\Theta_{j}:\left.(V \otimes B)\right|_{\widetilde{U}_{j}} \rightarrow \widetilde{U}_{j} \times B^{r} .
$$


Take relatively compact open subsets $U_{j} \subset \subset \widetilde{U}_{j}$ such that $U_{1}, \ldots, U_{N}$ is still an open covering of $X$. Set

$$
\omega_{j}=\Theta_{j}\left(\left.\omega \cap(V \otimes B)\right|_{\bar{U}_{j}}\right) .
$$

Then each $\omega_{j}$ is a compact subset of $\bar{U}_{j} \times B^{r}$. Now we consider an arbitrary $\delta>0$. As $\widetilde{\omega}:=\omega_{1} \cup \ldots \cup \omega_{N}$ is compact, then we can choose a finite number of points $\left(\zeta_{\nu}, b_{\nu}\right) \in X \times B^{r}, v=1, \ldots, m$, such that, for each $(\zeta, b) \in \widetilde{\omega}$, there exists an index $v \in\{1, \ldots, m\}$ with $d\left((\zeta, b),\left(\zeta_{\nu}, b_{v}\right)\right)<\delta$ and therefore, by definition of $d,\left\|b-b_{v}\right\|_{B^{r}}<\delta$. Let $F$ be the subspace of $B$ spanned by the components of the vectors $b_{v}$ (the dimension of $F$ is $\leq m r$ ). Then $F^{r}$ contains the vectors $b_{v}$ and, hence, for each $(\zeta, b) \in \widetilde{\omega}$, we get

$$
\inf _{v \in F^{r}}\|b-v\|_{B^{r}}<\delta
$$

Since $\Theta_{j}\left((V \otimes F)_{\zeta}\right)=\{\zeta\} \times F^{r}$ for $\zeta \in \bar{U}_{j}$, this implies (2.16) if $\delta$ is chosen sufficiently small.

Proposition 2.8. Let $V, V^{\prime}$ and $V^{\prime \prime}$ be $\mathcal{C}^{\infty}$ vector bundles over a compact $\mathcal{C}^{\infty}$ manifold $X, B$ a Banach space and $0<\alpha<1$. Let $K: X \rightarrow \operatorname{Hom}\left(V \otimes B, V^{\prime} \otimes B\right)$ be a section which is fiber compact and continuous with respect to the operator norm topology. Let $A: \mathcal{C}^{0}\left(X, V^{\prime}\right) \rightarrow \mathcal{C}^{\alpha}\left(X, V^{\prime \prime}\right)$ be a bounded linear operator, and let

$$
A_{B}: \mathcal{C}^{0}\left(X, V^{\prime} \otimes B\right) \longrightarrow \mathcal{C}^{\alpha}\left(X, V^{\prime \prime} \otimes B\right)
$$

be the bounded linear operator with $A_{B}=A \otimes$ id on $\mathcal{C}^{0}\left(X, V^{\prime}\right) \otimes B$ (Proposition 2.2). Then $A_{B} K$ is compact as operator between $\mathcal{C}^{0}(X, V \otimes B)$ and $\mathcal{C}^{0}\left(X, V^{\prime \prime} \otimes B\right)$.

Proof. Fix some fiber norms on $V \otimes B, V^{\prime} \otimes B$ and $V^{\prime \prime} \otimes B$ which we all denote by $\|\cdot\|$. Consider an arbitrary sequence $\left(f_{j}\right)_{j \in \mathbb{N}}$ in $\mathcal{C}^{0}(X, V \otimes B)$ with

$$
C:=\sup _{j \in \mathbb{N}, \zeta \in X}\left\|f_{j}(\zeta)\right\|<\infty
$$

as well as an arbitrary $\varepsilon>0$. It is sufficient to find a sequence $\left(f_{j}^{\prime \prime}\right)_{j \in \mathbb{N}}$ in $\mathcal{C}^{0}\left(X, V^{\prime \prime} \otimes B\right)$ with

$$
\max _{\zeta \in X}\left\|A_{B} K f_{j}(\zeta)-f_{j}^{\prime \prime}(\zeta)\right\| \leq \varepsilon \quad \text { for all } j \in \mathbb{N}
$$

such that $\left(f_{j}^{\prime \prime}\right)_{j \in \mathbb{N}}$ has a uniformly convergent subsequence. 
Let $\left\|A_{B}\right\|$ be the operator norm of $A_{B}$ as an operator between the spaces $\mathcal{C}^{0}\left(X, V^{\prime} \otimes B\right)$ and $\mathcal{C}^{0}\left(X, V^{\prime \prime} \otimes B\right)$ endowed with the maximum norms with respect to the fiber norms fixed in the beginning of the proof. By Lemma 2.4, the set

$$
\omega:=\left\{K(\zeta) f_{j}(\zeta) \mid \zeta \in X, j \in \mathbb{N}\right\}
$$

is precompact in $V^{\prime} \otimes B$. Hence, by Lemma 2.7, there is a finite dimensional subspace $F$ of $B$ such that

$$
\inf _{w \in\left(V^{\prime} \otimes F\right)_{\zeta}}\left\|K(\zeta) f_{j}(\zeta)-w\right\|<\frac{\varepsilon}{\left\|A_{B}\right\|} \quad \text { for all } \zeta \in X \text { and } j \in \mathbb{N} .
$$

Therefore, by lemma 2.6, we can find a sequence $\left(f_{j}^{\prime}\right)_{j \in \mathbb{N}}$ in $\mathcal{C}^{0}\left(X, V^{\prime} \otimes F\right)$ such that

$$
\max _{\zeta \in X}\left\|K(\zeta) f_{j}(\zeta)-f_{j}^{\prime}(\zeta)\right\|<\frac{\varepsilon}{\left\|A_{B}\right\|} \quad \text { for all } j \in \mathbb{N} .
$$

Set $f_{j}^{\prime \prime}=A_{B} f_{j}^{\prime}$. Then (2.18) is clear, by (2.21) and the definition of $\left\|A_{B}\right\|$. Moreover, as $A_{B}$ is the tensor product of $A$ and the identical map of $B, A_{B}\left(C^{0}\left(X, V^{\prime} \otimes\right.\right.$ $F)$ ) is contained in $\mathcal{C}^{\alpha}\left(X, V^{\prime \prime} \otimes F\right)$. Hence $A_{B}$ is a bounded linear operator from $\mathcal{C}^{0}\left(X, V^{\prime} \otimes F\right)$ to $\mathcal{C}^{\alpha}\left(X, V^{\prime \prime} \otimes F\right)$, and from (2.17) and the continuity of $K$ it follows that the sequence $\left(f_{j}^{\prime}\right)_{j \in \mathbb{N}}$ is bounded in $\mathcal{C}^{0}\left(X, V^{\prime} \otimes F\right)$. Therefore, the sequence $\left(f_{j}^{\prime \prime}\right)_{j \in \mathbb{N}}$ is bounded in $\mathcal{C}^{\alpha}\left(X, V^{\prime \prime} \otimes F\right)$. Since $V^{\prime \prime} \otimes F$ has finite rank, this implies, by Ascoli's theorem, that $\left(f_{j}^{\prime \prime}\right)_{j \in \mathbb{N}}$ has a uniformly convergent subsequence.

\section{3. $\bar{\partial}$ for continuous Banach-valued functions}

Let $E$ be a holomorphic Banach bundle over a complex manifold $X$. Then we denote by $\Lambda^{p, q} T_{X}^{*}$ the complex vector bundle of $(p, q)$-forms on $X$. The sections of $\Lambda^{p, q} T_{X}^{*} \otimes E$ will be called $E$-valued $(p, q)$-forms. Set $\mathcal{C}_{p, q}^{k}(X, E)=$ $\mathcal{C}^{k}\left(X, \Lambda^{p, q} T_{X}^{*} \otimes E\right)$ for $k \in \mathbb{N} \cup\{\infty\}$ and $\mathcal{C}_{p, q}^{k+\alpha}(X, E)=\mathcal{C}^{k+\alpha}\left(X, \Lambda^{p, q} T_{X}^{*} \otimes E\right)$ if $k \in \mathbb{N}$ and $0<\alpha<1$. If $B$ is a Banach space, then we write $\Lambda^{p, q} T_{X}^{*} \otimes B$ instead of $\Lambda^{p, q} T_{X}^{*} \otimes(X \times B)$ and $\mathcal{C}_{p, q}^{\lambda}(X, B)$ instead of $\mathcal{C}_{p, q}^{\lambda}(X, X \times B), 0 \leq \lambda \leq \infty$.

Now let $X$ be an open subset of $\mathbb{C}^{n}$. Denote by $\mathcal{I}_{p}$ the set of strictly increasing $p$-tuples of length $p$ in $\{1, \ldots, n\}$. Let $B$ be a Banach space and $f$ a $B$-valued $(p, q)$-form on $X$. Then by the coefficients of $f$ we mean the maps $f_{I J}: X \rightarrow B$, $I \in \mathcal{I}_{p}, J \in \mathcal{I}_{q}$, with $f=\sum_{I \in \mathcal{I}_{p}, J \in \mathcal{I}_{q}} f_{I J} d z_{I} \wedge d \bar{z}_{J}$. If $H$ is an End $B$-valued map on $X$, then we denote by $H f$ the form with coefficients $H \circ f_{I J}$.

Definition 3.1. Let $X$ be an open subset of $\mathbb{C}^{n}$ and $B$ a Banach space. For $f \in$ $\mathcal{C}_{p, q}^{0}(X, B)$, we say that $\bar{\partial} f$ is continuous if there exists $g \in \mathcal{C}_{p, q+1}^{0}(X, B)$ with

$$
\int_{X} \bar{\partial} \psi \wedge f=(-1)^{p+q} \int_{X} \psi \wedge g
$$


for all scalar $\psi \in \mathcal{C}_{n-p, n-q-1}^{\infty}(X, \mathbb{C})$ with compact support. Then $g$ is uniquely determined and will be denoted by $\bar{\partial} f$. If $\bar{\partial} f=0$, then $f$ will be called $\bar{\partial}$-closed. Set $\widetilde{\mathcal{C}}_{p, q}^{0}(X, B)=\left\{f \in \mathcal{C}_{p, q}^{0}(X, B) \mid \bar{\partial} f\right.$ is continuous $\}$ and $\mathcal{Z}_{p, q}^{0}(X, B)=\{f \in$ $\left.\widetilde{\mathcal{C}}_{p, q}^{0}(X, B) \mid \bar{\partial} f=0\right\}$.

Note that $\bar{\partial}$ is closed as operator between the Fréchet spaces $\mathcal{C}_{p, q}^{0}(X, B)$ and $\mathcal{C}_{p, q+1}^{0}(X, B)$ if we take $\widetilde{\mathcal{C}}_{p, q}^{0}(X, B)$ as domain of definition.

Proposition 3.2. Let $X$ be an open subset of $\mathbb{C}^{n}, B$ a Banach space and $f \in$ $\widetilde{\mathcal{C}}_{p, q}^{0}(X, B)$. Then:

(i) For each complex valued $\mathcal{C}^{\infty}$-function $\chi$ on $X, \chi f$ belongs to $\widetilde{\mathcal{C}}_{p, q}^{0}(X, B)$ and $\bar{\partial}(\chi f)=\bar{\partial} \chi \wedge f+\chi \bar{\partial} f$.

(ii) For each holomorphic map $H: X \rightarrow$ End $B, H f$ belongs to $\widetilde{\mathcal{C}}_{p, q}^{0}(X, B)$ and $\bar{\partial} H f=H \bar{\partial} f$.

Proof. To prove (i), for $\psi \in \mathcal{C}_{n-p, n-q-1}^{\infty}(X, \mathbb{C})$ with compact support, we compute:

$$
\begin{aligned}
\int_{X} \bar{\partial} \psi \wedge \chi f & =\int_{X} \chi \bar{\partial} \psi \wedge f=\int_{X} \bar{\partial}(\chi \psi) \wedge f-\int_{X} \bar{\partial} \chi \wedge \psi \wedge f \\
& =(-1)^{p+q}\left(\int_{X} \chi \psi \wedge \bar{\partial} f+\int_{X} \psi \wedge \bar{\partial} \chi \wedge f\right) \\
& =(-1)^{p+q} \int_{X} \psi \wedge(\chi \bar{\partial} f+\bar{\partial} \chi \wedge f) .
\end{aligned}
$$

Now we prove (ii). Let $g=\bar{\partial} f$. First consider the case when $H$ is a constant map. Then, for each $\psi \in \mathcal{C}_{n-p, n-q-1}^{\infty}(X, \mathbb{C})$ with compact support,

$$
\int_{X} \bar{\partial} \psi \wedge H f=H\left(\int_{X} \bar{\partial} \psi \wedge f\right)=H\left((-1)^{p+q} \int_{X} \psi \wedge g\right)=(-1)^{p+q} \int_{X} \psi \wedge H g .
$$

Now consider the general case. Since the assertion is local, we may assume that $H$ is given by an uniformly convergent power series $H(z)=\sum z^{I} H_{I}$. Since the assertion is already proved for constant maps and by part (i), it follows that each $z^{I} H_{I} f$ belongs to $\widetilde{\mathcal{C}_{p, q}^{0}}(X, B)$ and $\bar{\partial}\left(z^{I} H_{I} f\right)=z^{I} H_{I} g$. Since both the series $\sum z^{I} H_{I} f$ and the series $\sum z^{I} H_{I} g$ converge uniformly on the compact subsets of $X$ and $\bar{\partial}$ is closed, this implies the assertion.

Definition 3.3. Let $E$ be a holomorphic Banach bundle over a complex manifold $X$. If $f \in \mathcal{C}_{p, q}^{0}(X, E)$, then we say that $\bar{\partial} f$ is continuous if there exists a form $g \in \mathcal{C}_{p, q+1}^{0}(X, E)$ such that $\bar{\partial} \Theta f=\Theta g$ for any local holomorphic trivialization $\Theta:\left.E\right|_{U} \rightarrow U \times B$ of $E$. We set $\bar{\partial} f=g$ in this case. Denote by $\widetilde{\mathcal{C}}_{p, q}^{0}(X, E)$ 
the space of all $f \in \mathcal{C}_{p, q}^{0}(X, E)$ with continuous $\bar{\partial} f$. Set $\mathcal{Z}_{p, q}^{0}(X, E)=\{f \in$ $\left.\widetilde{\mathcal{C}}_{p, q}^{0}(X, E) \mid \bar{\partial} f=0\right\}$. Further, we denote by $\mathcal{O}(X, E)$ the space of global holomorphic sections of $E$.

Remark 3.4. By Proposition 3.2 (ii) it is not necessary to verify $\bar{\partial} \Theta f=\Theta g$ for any local holomorphic trivialization over any coordinate chart but it suffices to do this for at least one local holomorphic trivialization over at least one coordinate neighborhood of any given point of $X$.

Proposition 3.5. Let $E$ be a holomorphic Banach bundle over an $n$-dimensional complex manifold $X$. Then $\mathcal{O}(X, E)=\mathcal{Z}_{0,0}^{0}(X, E)$.

Proof. Since the assertion is local, it is sufficient to consider an open set $X \subseteq \mathbb{C}^{n}$, a Banach space $B$ and a continuous map $f: X \rightarrow B$ with $\bar{\partial} f=0$, and to prove that $f$ is holomorphic. Since $\bar{\partial} f=0$, we have $\int_{X}(\bar{\partial} \psi) f=0$ for all $\psi \in \mathcal{C}_{n, n-1}^{\infty}(X, \mathbb{C})$ with compact support. Let $B^{*}$ be the dual space of $B$. Then this implies that $\int_{X}(\Phi \circ f) \bar{\partial} \psi=0$ for each $\Phi \in B^{*}$ and all $\psi \in \mathcal{C}_{n, n-1}^{\infty}(X, \mathbb{C})$ with compact support. Therefore $\bar{\partial}(\Phi \circ f)=0$ in the sense of distributions for each $\Phi \in B^{*}$. Hence (since the assertion of the proposition is well-known for $B=\mathbb{C}$ ) $\Phi \circ f$ is holomorphic for all $\Phi \in B^{*}$. As $f$ is continuous, this completes the proof.

Proposition 3.6. Let $E$ be a holomorphic Banach bundle over an $n$-dimensional complex manifold $X$ and $0 \leq p, q \leq n$. Then, for each $f \in \widetilde{\mathcal{C}}_{p, q}^{0}(X, E)$, there exists a sequence $\left(f_{\nu}\right)_{v \in \mathbb{N}}$ in $\mathcal{C}_{p, q}^{\infty}(X, E)$ such that, uniformly on the compact sets in $X$, both $\left(f_{v}\right)_{v \in \mathbb{N}}$ converges to $f$ and $\left(\bar{\partial} f_{v}\right)_{v \in \mathbb{N}}$ converges to $\bar{\partial} f$.

Proof. By Proposition 3.2 (i), each form in $f \in \widetilde{\mathcal{C}}_{p, q}^{0}(X, E)$ can be written as a locally finite sum $f=\sum_{j} f_{j}$ of forms $f_{j} \in \widetilde{\mathcal{C}}_{p, q}^{0}(X, E)$ such that the support of each $f_{j}$ is compact and contained in a coordinate neighborhood where $E$ is trivial. Therefore we may assume that $f \in \widetilde{\mathcal{C}}_{p, q}^{0}(X, B)$ for some Banach space $B$.

Let $d \lambda$ be the Euclidean volume form on $\mathbb{C}^{n}$. Fix a non-negative $\mathcal{C}^{\infty}$-function $\varphi$ on $\mathbb{C}^{n}$ with $\int_{\mathbb{C}^{n}} \varphi d \lambda=1$ and $\varphi(\zeta)=0$ if $|\zeta| \geq 1$. Set $\varphi_{\varepsilon}(\zeta)=\varepsilon^{-2 n} \varphi(\zeta / \varepsilon)$ for $\zeta \in \mathbb{C}^{n}$ and $\varepsilon>0$. Let for $\zeta \in \mathbb{C}^{n}$ define

$$
t_{\zeta}: \mathbb{C}^{n} \ni z \rightarrow z-\zeta \in \mathbb{C}^{n}
$$

and for any $B$-valued continuous form $u$ on $\mathbb{C}^{n}$

$$
T_{\varepsilon} u=\int_{\mathbb{C}^{n}} \varphi_{\varepsilon}(\zeta) t_{\zeta}^{*} u d \lambda(\zeta)
$$

Then $T_{\varepsilon} u$ is $\mathcal{C}^{\infty}$, since its coefficients are obtained from those of $u$ by a convolution with $\varphi_{\varepsilon}$; and $T_{\varepsilon} u \rightarrow u$, uniformly on the compact sets, when $\varepsilon \rightarrow 0$, by the same reason. As $T_{\varepsilon}$ commutes with $\bar{\partial}$ on $\widetilde{\mathcal{C}}_{p, q}(X, B)$, one takes $f_{v}=T_{\mathcal{E}_{v}} f$, with a sequence $\varepsilon_{v} \rightarrow 0$. 
Proposition 3.7. Let $X$ be a convex open subset of $\mathbb{C}^{n}, U$ a relatively compact open subset of $X, B$ a complex Banach space and $0 \leq p \leq n$. Then there exist linear operators

$$
A_{r}: \mathcal{C}_{p, r}^{0}(X, B) \longrightarrow \mathcal{C}_{p, r-1}^{0}(U, B), \quad 1 \leq r \leq n,
$$

with $A_{r}\left(\widetilde{\mathcal{C}}_{p, r}^{0}(X, B)\right) \subseteq \widetilde{\mathcal{C}}_{p, r-1}^{0}(U, B)$ such that (setting $A_{n+1}=0$ )

$$
\bar{\partial} A_{r} f+A_{r+1} \bar{\partial} f=\left.f\right|_{U} \quad \text { for all } f \in \widetilde{\mathcal{C}}_{p, r}^{0}(X, B) .
$$

Moreover, if $k \in \mathbb{N} \cup\{\infty\}, 0 \leq \alpha<1$ and $1 \leq r \leq n$, then $A_{r}\left(\mathcal{C}_{p, r}^{k}(X, B)\right) \subseteq$ $\mathcal{C}_{p, r-1}^{k+\alpha}(X, B)$ and $A_{r}$ is continuous as an operator from $\mathcal{C}_{p, r}^{k}(X, B)$ to $\mathcal{C}_{p, r-1}^{k+\alpha}(U, B)$.

Proof. If $B=\mathbb{C}$, then the assertion is well known, ${ }^{3}$ i.e. we have linear operators $A_{r}^{\mathbb{C}}: \mathcal{C}_{p, r}^{0}(X, \mathbb{C}) \rightarrow \bigcap_{0<\alpha<1} \mathcal{C}_{p, r-1}^{\alpha}(U, \mathbb{C})$, which are $\mathcal{C}^{k}-\mathcal{C}^{k+\alpha}$-continuous for $0<\alpha<1$ and each $k \in \mathbb{N} \cup\{\infty\}$, such that

$$
\bar{\partial} A_{r}^{\mathbb{C}} \varphi+A_{r+1}^{\mathbb{C}} \bar{\partial} \varphi=\varphi \quad \text { for all } \varphi \in \widetilde{\mathcal{C}}_{p, r}^{0}(X, \mathbb{C}) .
$$

Then, by Proposition 2.2, there are linear operators $A_{r}: \mathcal{C}_{p, r}^{0}(X, B) \rightarrow \bigcap_{0<\alpha<1}$ $\mathcal{C}_{p, r-1}^{\alpha}(U, B)$, which are $\mathcal{C}^{k}-\mathcal{C}^{k+\alpha}$-continuous for $0<\alpha<1$ and each $k \in \mathbb{N}$, such that

$$
A_{r} \sum_{j=1}^{N} \varphi_{j} b_{j}=\sum_{j=1}^{N} A_{r}^{\mathbb{C}}\left(\varphi_{j}\right) b_{j} \quad \text { if } \varphi_{j} \in \mathcal{C}_{p, r}^{0}(X, \mathbb{C}), b_{j} \in B, N<\infty .
$$

It remains to prove (3.2). Let $f \in \widetilde{\mathcal{C}}_{p, r}^{0}(X, B)$ be given. By Propositions 3.6 and 2.1 there exists a sequence $f_{v} \in \mathcal{C}_{p, r}^{\infty}(X, \mathbb{C}) \otimes B$ such that, in the $\mathcal{C}^{0}$ topology, $\lim f_{v}=$ $f$ and $\lim \bar{\partial} f_{v}=\bar{\partial} f$. From (3.4) and (3.3) it follows $\bar{\partial} A_{r} f_{v}+A_{r+1} \bar{\partial} f_{v}=f_{v}$ for all $v$. Passing to the limit we obtain (3.2).

Theorem 3.8. Let $X$ be an n-dimensional complex manifold, $E \rightarrow X$ a holomorphic Banach bundle. Then, for $0 \leq r \leq n$, there are isomorphisms

$$
H^{r}(X, E) \cong \frac{\mathcal{Z}_{0, r}^{\infty}(X, E)}{\bar{\partial} \mathcal{C}_{0, r-1}^{\infty}(X, E)} \cong \frac{\mathcal{Z}_{0, r}^{0}(X, E)}{\bar{\partial} \widetilde{\mathcal{C}}_{0, r-1}^{0}(X, E)},
$$

where the second isomorphism is induced by the identity map $\mathcal{Z}_{0, r}^{\infty}(X, E) \rightarrow \mathcal{Z}_{0, r}^{0}$ $(X, E)$ and $\mathcal{C}_{0,-1}^{\infty}(X, E):=\widetilde{\mathcal{C}}_{0,-1}^{0}(X, E):=0$.

${ }^{3}$ Indeed, we may assume that also $U$ is convex. Moreover, we may assume that the boundary of $U$ is smooth. Then, for example, we can take the Henkin operators (see, e.g., Corollary 1.12.2 in [6] and, for the existence of the holomorphic "Leray map" required there, Corollary 2.1.4 in [6].) 
Proof. By Propositions 3.2 (i), 3.5 and 3.7, the sequences of sheaves

$$
0 \longrightarrow \mathcal{O}^{E} \longrightarrow \widetilde{\mathcal{C}}_{0,1}^{0}(\cdot, E) \stackrel{\bar{\partial}}{\longrightarrow} \widetilde{\mathcal{C}}_{0,1}^{0}(\cdot, E) \stackrel{\bar{\partial}}{\longrightarrow} \ldots \stackrel{\bar{\partial}}{\longrightarrow} \widetilde{\mathcal{C}}_{0, n}^{0}(\cdot, E) \longrightarrow 0
$$

and

$$
0 \longrightarrow \mathcal{O}^{E} \longrightarrow \mathcal{C}_{0,1}^{\infty}(\cdot, E) \stackrel{\bar{\partial}}{\longrightarrow} \mathcal{C}_{0,1}^{\infty}(\cdot, E) \stackrel{\bar{\partial}}{\longrightarrow} \ldots \stackrel{\bar{\partial}}{\longrightarrow} \mathcal{C}_{0, n}^{\infty}(\cdot, E) \longrightarrow 0
$$

are fine resolutions of $\mathcal{O}^{E}$. Therefore the assertion follows from the de Rham-Weil isomorphism.

\section{A global homotopy formula}

Theorem 4.1. Let $X$ be an $n$-dimensional compact complex manifold, $V \rightarrow X a$ holomorphic vector bundle and $0 \leq q \leq n$ such that

$$
H^{0, q}(X, V)=0 .
$$

Further let $0<\alpha<1$. Then there exist continuous linear operators $A_{r}: \mathcal{C}_{0, r}^{0}(X, V) \rightarrow$ $\mathcal{C}_{0, r-1}^{\alpha}(X, V), r=q, q+1,\left(A_{0}:=A_{n+1}:=0\right)$ with

$$
\bar{\partial} A_{q} f+A_{q+1} \bar{\partial} f=f
$$

for all $f \in \mathcal{C}_{0, q}^{0}(X, V)$ such that $\bar{\partial} f$ is also continuous.

This section is devoted to the proof of this theorem. We will deduce it from the Koppelman formula by means of a general functional analytic local-global construction described in [8]. For convenience of the reader we repeat this construction adapted to the situation considered here. $r \leq n$.

Let $\widetilde{\mathcal{C}}_{0, r}^{0}(X, V)$ be the space of all $f \in \mathcal{C}_{0, r}^{0}(X, V)$ with continuous $\bar{\partial} f, 0 \leq$

By means of the Koppelman formula and a partition of unity (see [7], Theorem 1.17, for the details), we obtain continuous linear operators $T_{r}: \mathcal{C}_{0, r}^{0}(X, V) \rightarrow$ $\mathcal{C}_{0, r-1}^{\alpha}(X, V)$ and $K_{r}: \mathcal{C}_{0, r}^{0}(X, V) \rightarrow \mathcal{C}_{0, r}^{\alpha}(X, V)$ such that (with $T_{n+1}:=0$ )

$$
f+K_{r} f=\bar{\partial} T_{r} f+T_{r+1} \bar{\partial} f \quad \text { for all } f \in \widetilde{\mathcal{C}}_{0, r}^{0}(X, V) \quad \text { and } \quad 1 \leq r \leq n .
$$

Applying $\bar{\partial}$ to (4.3), we get $\bar{\partial} f+\bar{\partial} K_{r} f=\bar{\partial} T_{r+1} \bar{\partial} f$, and replacing $f$ by $\bar{\partial} f$ and $r$ by $r+1$ in (4.3), we obtain (setting also $\left.K_{n+1}:=0\right) \bar{\partial} f+K_{r+1} \bar{\partial} f=\bar{\partial} T_{r+1} \bar{\partial} f$. Together this implies

$$
\bar{\partial} K_{r} f=K_{r+1} \bar{\partial} f \quad \text { for all } \quad f \in \widetilde{\mathcal{C}}_{0, r}^{0}(X, V) \quad \text { and } \quad 1 \leq r \leq n .
$$


Let $1 \leq r \leq n$. Set $\mathcal{B}_{r}=\bar{\partial} \widetilde{\mathcal{C}}_{0, r-1}^{0}(X, V)$. Note that $\mathcal{B}_{r}$ is a closed subspace of $\mathcal{Z}_{0, r}^{0}(X, V)$ (with respect to the max-norm), because it is of finite codimension and the image of a closed linear operator. Set $N_{r}:=\mathrm{id}+\left.K_{r}\right|_{\mathcal{B}_{r}}$. Since $K_{r}\left(\mathcal{B}_{r}\right) \subseteq$ $\mathcal{B}_{r}$ (as it follows from (4.3)), $N_{r}$ is an endomorphism of $\mathcal{B}_{r}$. Let $\operatorname{Ker} N_{r}$ be the kernel, and $\operatorname{Im} N_{r}$ the image of $N_{r} . N_{r}-\mathrm{id}=\left.K_{r}\right|_{\mathcal{B}_{r}}$ is continuous as an operator between $\mathcal{B}_{r}$ and $\mathcal{C}_{0, r}^{\alpha}(X, V)$ and, therefore, by Ascoli's theorem, compact as an endomorphism of $\mathcal{B}_{r}$. Hence $N_{r}$ is a Fredholm endomorphism of $\mathcal{B}_{r}$ with index zero. Let $m_{r}:=\operatorname{dim} \operatorname{Ker} N_{r}=\operatorname{dim} \mathcal{B}_{r} / \operatorname{Im} N_{r}$. Take a basis $b_{1}^{r}, \ldots, b_{m_{r}}^{r}$ of $\operatorname{Ker} N_{r}$ and forms $g_{1}^{r}, \ldots, g_{m_{r}}^{r} \in \mathcal{B}_{r}$ such that $\mathcal{B}_{r}$ is spanned by $\operatorname{Im} N_{r} \cup\left\{g_{1}^{r}, \ldots, g_{m_{r}}^{r}\right\}$. As $\mathcal{C}_{0, r-1}^{\infty}(X, V)$ is dense in $\widetilde{\mathcal{C}}_{0, r-1}^{0}(X, V)$ with respect to the graph norm of $\bar{\partial}$, we may assume that $g_{v}^{r} \in \bar{\partial} \mathcal{C}_{0, r-1}^{\infty}(X, V)$. Choose $u_{v}^{r-1} \in \mathcal{C}_{0, r-1}^{\infty}(X, V)$ with

$$
\bar{\partial} u_{v}^{r-1}=g_{v}^{r}, \quad 1 \leq v \leq m_{r} .
$$

Let $V^{*}$ be the dual bundle of $V$. Take forms $\varphi_{1}^{r}, \ldots, \varphi_{m_{r}}^{r} \in C_{n, n-r}^{\infty}\left(X, V^{*}\right)$ with

$$
\int_{X} b_{v}^{r} \wedge \varphi_{\mu}^{r}=\delta_{v \mu} \quad \text { (Kronecker symbol), } \quad 1 \leq \nu, \mu \leq m_{r} .
$$

Define continuous linear operators of finite rank $S_{r}: \mathcal{C}_{0, r}^{0}(X, V) \rightarrow \mathcal{C}_{0, r-1}^{\infty}(X, V)$ and $K_{r}^{\prime}, K_{r}^{\prime \prime}: \mathcal{C}_{0, r}^{0}(X, V) \rightarrow \mathcal{C}_{0, r}^{\infty}(X, V)$ by

$$
S_{r} f=\sum_{\nu=1}^{m_{r}}\left(\int_{X} f \wedge \varphi_{\nu}^{r}\right) u_{\nu}^{r-1}, \quad K_{r}^{\prime} f=\sum_{\nu=1}^{m_{r}}\left(\int_{X} f \wedge \varphi_{\nu}^{r}\right) g_{v}^{r}
$$

and

$$
K_{r}^{\prime \prime} f=(-1)^{p+r+1} \sum_{\nu=1}^{m_{r}}\left(\int_{X} f \wedge \bar{\partial} \varphi_{\nu}^{r}\right) u_{\nu}^{r}
$$

Then, by (4.5),

$$
K_{r}^{\prime} f=\bar{\partial} S_{r} f \quad \text { for all } \quad f \in \mathcal{C}_{0, r}^{0}(X, V) \quad \text { and } \quad 1 \leq r \leq n,
$$

and, by Stokes' theorem, we obtain (setting $S_{n+1}:=0$ )

$$
K_{r}^{\prime \prime} f=S_{r+1} \bar{\partial} f \quad \text { for all } f \in \widetilde{\mathcal{C}}_{0, r}^{0}(X, V) \quad \text { and } \quad 1 \leq r \leq n .
$$

Set

$$
\tilde{A}_{r}=T_{r}+S_{r} \quad \text { and } \quad M_{r}=\mathrm{id}+K_{r}+K_{r}^{\prime}+K_{r}^{\prime \prime} \quad \text { for } 1 \leq r \leq n .
$$

It follows from (4.3), (4.7) and (4.8) that (with $\widetilde{A}_{n+1}:=0$ )

$$
\bar{\partial} \widetilde{A}_{r} f+\widetilde{A}_{r+1} \bar{\partial} f=M_{r} f \quad \text { for all } \quad f \in \widetilde{\mathcal{C}}_{0, r}^{0}(X, V) \quad \text { and } \quad 1 \leq r \leq n .
$$

It follows (setting also $M_{n+1}:=0$ )

$$
\bar{\partial} M_{r} f=M_{r+1} \bar{\partial} f \quad \text { for all } f \in \widetilde{\mathcal{C}}_{0, r}(X, V) \text { and } 1 \leq r \leq n .
$$


Lemma 4.2. For $1 \leq r \leq n$ we have:

(i) $M_{r}$ is a Fredholm endomorphism of $\mathcal{C}_{0, r}^{0}(X, V)$ with index zero;

(ii) $\left.M_{r}\right|_{\mathcal{B}_{r}}$ is an isomorphism of $\mathcal{B}_{r}$.

Proof. Since $K_{r}+K_{r}^{\prime}+K_{r}^{\prime \prime}$ is continuous as operator from $\mathcal{C}_{0, r}^{0}(X, V)$ to $\mathcal{C}_{0, r}^{\alpha}(X, V)$, it follows from Ascoli's theorem that $M_{r}-\mathrm{id}=K_{r}+K_{r}^{\prime}+K_{r}^{\prime \prime}$ is compact as endomorphism of $\mathcal{C}_{0, r}^{0}(X, V)$. This proves part (i). Further, $K_{r}^{\prime}$ is a map of rank $m_{r}=\operatorname{dim} \operatorname{Ker} N_{r}$ which maps $\operatorname{Ker} N_{r}$ isomorphically onto a complement of $\operatorname{Im} N_{r}$ in $\mathcal{B}_{r}$ (by (4.6)). Hence $N_{r}+\left.K_{r}^{\prime}\right|_{\mathcal{B}_{r}}$ is an isomorphism of $\mathcal{B}_{r}$. This proves part (ii), because $K_{r}^{\prime \prime}$ vanishes on $\mathcal{B}_{r}$ and therefore $\left.M_{r}\right|_{\mathcal{B}_{r}}=N_{r}+\left.K_{r}^{\prime}\right|_{\mathcal{B}_{r}}$.

Set $h_{r}=\operatorname{dim} H^{0, r}(X, V)$. Then, by regularity of $\bar{\partial}$, we can find an $m_{r^{-}}$ dimensional subspace $\Lambda_{r}$ of $\mathcal{Z}_{0, r}^{\infty}(X, V)$ such that $\mathcal{Z}_{0, r}^{0}(X, V)=\mathcal{B}_{r} \oplus \Lambda_{r}$ (direct sum). Choose a basis $\lambda_{1}^{r}, \ldots, \lambda_{h_{r}}^{r}$ of $\Lambda_{r}$. Moreover, by Serre duality, for each $r$, we can find $\gamma_{1}^{r}, \ldots, \gamma_{h_{r}}^{r} \in \mathcal{Z}_{n, n-r}^{\infty}\left(X, V^{*}\right)$ with

$$
\int_{X} \gamma_{\nu}^{r} \wedge \lambda_{\mu}^{r}=\delta_{v \mu} \quad \text { for } \quad 1 \leq v, \mu \leq m_{r} \quad \text { and } \quad 1 \leq r \leq n .
$$

Define projections $P_{r}$ from $\mathcal{C}_{0, r}^{0}(X, V)$ onto $\Lambda_{r}, 1 \leq r \leq n$, by

$$
P_{r} f=\sum_{\nu=1}^{h_{r}}\left(\int_{X} \gamma_{\nu}^{r} \wedge f\right) \lambda_{\nu}^{r} .
$$

Since the forms $\gamma_{v}^{r}$ and $\lambda_{v}^{r}$ are $\bar{\partial}$-closed, then

$$
\bar{\partial} P_{r} f=0 \quad \text { for all } f \in \widetilde{\mathcal{C}}_{0, r}^{0}(X, V) \text { and } 1 \leq r \leq n,
$$

and

$$
P_{r} \bar{\partial} f=0 \quad \text { for all } f \in \widetilde{\mathcal{C}}_{0, r-1}^{0}(X, V) \text { and } 1 \leq r \leq n .
$$

Together with (4.10) this implies that (with $P_{n+1}:=0$ )

$$
\bar{\partial}\left(M_{r}+P_{r}\right) f=\left(M_{r+1}+P_{r+1}\right) \bar{\partial} f \quad \text { for all } f \in \widetilde{\mathcal{C}}_{0, r}^{0}(X, V) \text { and } 1 \leq r \leq n .
$$

Lemma 4.3. $\left(M_{r}+P_{r}\right)\left(\widetilde{\mathcal{C}}_{0, r}^{0}(X, V)\right)=\widetilde{\mathcal{C}}_{0, r}^{0}(X, V)$ for $1 \leq r \leq n$.

Proof. " $\subseteq$ " follows from $K_{r}\left(\widetilde{\mathcal{C}}_{r, r}^{0}(X, V)\right) \subseteq \widetilde{\mathcal{C}}_{0, r}^{0}(X, V)$ (true by (4.4)) and the fact that the values of $K_{r}^{\prime}, K_{r}^{\prime \prime}$ and $P_{r}$ are of class $\mathcal{C}^{\infty}$. To prove "卫", first note that

$$
\mathcal{Z}_{0, r}^{0}(X, V)=\mathcal{B}_{r} \oplus \Lambda_{r}=M_{r}\left(\mathcal{B}_{r}\right) \oplus \operatorname{Im} P_{r} \subseteq\left(M_{r}+P_{r}\right)\left(\widetilde{\mathcal{C}}_{0, r}^{0}(X, V)\right) .
$$

Now let $f \in \widetilde{\mathcal{C}}_{0, r}^{0}(X, V)$. Then $\bar{\partial} f \in \mathcal{B}_{r+1}=M_{r+1}\left(\mathcal{B}_{r+1}\right)$ (with $\mathcal{B}_{n+1}:=0$ ). Therefore and by (4.10), $\bar{\partial} f=M_{r+1} \bar{\partial} u=\bar{\partial} M_{r} u$ for some $u \in \widetilde{\mathcal{C}}_{0, r}^{0}(X, V)$. Hence $f-M_{r} u \in \mathcal{Z}_{0, r}^{0}(X, V)$ which yields, by (4.15), $f-M_{r} u \in\left(M_{r}+P_{r}\right)\left(\widetilde{\mathcal{C}}_{0, r}^{0}(X, V)\right)$. Since also $M_{r} u \in\left(M_{r}+P_{r}\right)\left(\widetilde{\mathcal{C}}_{0, r}^{0}(X, V)\right)$, it follows $f \in\left(M_{r}+P_{r}\right)\left(\widetilde{\mathcal{C}}_{0, r}^{0}(X, V)\right)$. 
Since $M_{r}$ is an index zero Fredholm endomorphism of $\mathcal{C}_{0, r}^{0}(X, V)$, also $M_{r}+$ $P_{r}$ is an index zero Fredholm endomorphism of $\mathcal{C}_{0, r}^{0}(X, V)$. As $\widetilde{\mathcal{C}}_{0, r}^{0}(X, V)$ is dense in $\mathcal{C}_{0, r}^{0}(X, V)$, this implies by Lemma 4.3 that $M_{r}+P_{r}$ is an isomorphism of $\mathcal{C}_{0, r}^{0}(X, V)$. From (4.14) and Lemma 4.3 follows

$$
\bar{\partial}\left(M_{r}+P_{r}\right)^{-1} f=\left(M_{r+1}+P_{r+1}\right)^{-1} \bar{\partial} f, \quad f \in \widetilde{\mathcal{C}}_{0, r}^{0}(X, V), \quad 1 \leq r \leq n .
$$

Set $A_{r}=\widetilde{A}_{r}\left(M_{r}+P_{r}\right)^{-1}$ for $1 \leq r \leq n$. Then, by (4.9) and (4.16),

$$
\bar{\partial} A_{r} f+A_{r+1} \bar{\partial} f=f-P_{r}\left(M_{r}+P_{r}\right)^{-1} f, \quad f \in \widetilde{\mathcal{C}}_{0, r}^{0}(X, V), \quad 1 \leq r \leq n .
$$

If $q \geq 1$ and therefore $P_{q}=0$ (by hypothesis (4.1)), this completes the proof of Theorem 4.1. Now let $q=0$ and $f \in \widetilde{\mathcal{C}}_{0,0}^{0}(X, V)$. We have to prove that

$$
A_{1} \bar{\partial} f=f .
$$

Since $\left.\left(M_{1}+P_{1}\right)\right|_{\mathcal{B}_{1}}=\left.M_{1}\right|_{\mathcal{B}_{1}}$ is an isomorphism of $\mathcal{B}_{1}$, we see in particular that $\left(M_{1}+P_{1}\right)^{-1} \bar{\partial} f \in \mathcal{B}_{1}$ and therefore $P_{1}\left(M_{1}+P_{1}\right)^{-1} \bar{\partial} f=0$. In view of (4.17) (with $r=1$ and $\bar{\partial} f$ instead of $f$ ), this implies $\bar{\partial} A_{1} \bar{\partial} f=\bar{\partial} f-P_{1}\left(M_{1}+P_{1}\right)^{-1} \bar{\partial} f=$ $\bar{\partial} f$, i.e. $A_{1} \bar{\partial} f-f \in H^{0,0}(X, V)$. Since, by hypthesis, $H^{0,0}(X, V)=0$, this means (4.18).

\section{Proof of Theorem 1.2}

Let $B$ be the characteristic fiber of $E$. Fix $0<\alpha<1$. In view of Theorem 3.8 we have to prove that

$$
\operatorname{dim} \frac{\mathcal{Z}_{0, q}^{0}(X, V \otimes E)}{\bar{\partial} \widetilde{\mathcal{C}}_{0, q-1}^{0}(X, V \otimes E)}<\infty
$$

and

$$
\mathcal{Z}_{0, q}^{0}(X, V \otimes E)=\bar{\partial} \widetilde{\mathcal{C}}_{0, q-1}^{0}(X, V \otimes E) \quad \text { if } E \text { is trivial. }
$$

Using Propositions 2.2, 3.6 and 2.1 in the same way as in the proof of the local homotopy formula of Proposition 3.7, from Theorem 4.1 we obtain continuous linear operators $A_{r}: \mathcal{C}_{0, r}^{0}(X, V \otimes B) \rightarrow \mathcal{C}_{0, r-1}^{\alpha}(X, V \otimes B), r=q, q+1,\left(A_{0}:=0\right.$ and $\left.A_{n+1}:=0\right)$ such that $A_{r}\left(\widetilde{\mathcal{C}}_{0, r}^{0}(X, V \otimes B)\right) \subseteq \widetilde{\mathcal{C}}_{0, r-1}^{0}(X, V \otimes B)$ and

$$
\bar{\partial} A_{q} f+A_{q+1} \bar{\partial} f=f \quad \text { for all } f \in \widetilde{\mathcal{C}}_{0, q}^{0}(X, V \otimes B) .
$$

First note that this implies (5.2) and that we did not use the compact approximation property of $B$ for this.

Now we prove (5.1). Fix some norm $\|\cdot\|$ in $B$. The same notation $\|\cdot\|$ will be used for the fiber norm canonically defined by this norm in the trivial bundle $X \times B$. 
Further we fix some fiber norms in each of the bundles $V, E$ and $\Lambda^{0, r} T_{X}^{*} \otimes V \otimes B$, $0 \leq r \leq n$, which will be denoted also by $\|\cdot\|$. Since $E$ is of compact type, we can choose finite open coverings $U_{1}, \ldots, U_{N}$ and $U_{1}^{\prime}, \ldots, U_{j}^{\prime}$ of $X$ with $\bar{U}_{j} \subset \subset U_{N}^{\prime}$ for $j=1, \ldots, N$ as well as a family of holomorphic sections $\widetilde{\Phi}_{j}: U_{j}^{\prime} \longrightarrow \operatorname{Iso}(E, X \times$ $B)$ such that the sections

$$
\widetilde{\Gamma}_{j k}:=\widetilde{\Phi}_{j} \widetilde{\Phi}_{k}^{-1}-\mathrm{id}: U_{j}^{\prime} \longrightarrow X \times \text { End } B, \quad 1 \leq j, k \leq N
$$

are fiber compact. Then, by Lemma 2.4 , the set

$$
\left\{\widetilde{\Gamma}_{j k} b \mid b \in\left(\bar{U}_{j} \cap \bar{U}_{k}\right) \times B,\|b\|=1,1 \leq j, k \leq N\right\}
$$

is relatively compact in $\left(\bar{U}_{j} \cap \bar{U}_{k}\right) \times B$. Let $\varepsilon>0$ be a number which will be chosen below. As $B$ has the compact approximation property and (5.4) is relatively compact, we can find a compact linear operator $\widetilde{Q} \in$ End $B$ such that, if we denote the corresponding constant section of $X \times$ End $B$ also by $\widetilde{Q}$,

$$
\left\|\widetilde{\Gamma}_{j k} b-\widetilde{Q} \widetilde{\Gamma}_{j k} b\right\| \leq \varepsilon \quad \text { for all } b \in\left(\bar{U}_{j} \cap \bar{U}_{k}\right) \times B \text { with }\|b\|=1 .
$$

Setting $Q=\mathrm{id}_{V} \otimes \widetilde{Q}, P=\mathrm{id}_{V \otimes B}-Q, \Gamma_{j k}=\mathrm{id}_{V} \otimes \widetilde{\Gamma}_{j k}, \Phi_{j}=\mathrm{id}_{V} \otimes \widetilde{\Phi}_{j}$, we get holomorphic sections $P, Q: X \rightarrow$ End $(V \otimes B), \Phi_{j}: U_{j}^{\prime} \rightarrow \operatorname{Iso}(V \otimes E, V \otimes B)$ and $\Gamma_{j k}: U_{j}^{\prime} \cap U_{k}^{\prime} \rightarrow$ End $(V \otimes B)$. Then

$$
\Phi_{j} \Phi_{k}^{-1}=\mathrm{id}_{V \otimes B}+\Gamma_{j k} \quad \text { on } U_{j}^{\prime} \cap U_{k}^{\prime}, \quad 1 \leq j, k \leq N
$$

Hence $\Phi_{j}^{-1} P\left(\Phi_{j}-\Phi_{k}\right)=\Phi_{j}^{-1} P\left(\Phi_{j} \Phi_{k}^{-1}-\mathrm{id}_{V \otimes B}\right) \Phi_{k}=\Phi_{j}^{-1} P \Gamma_{j k} \Phi_{k}$ on $U_{j}^{\prime} \cap U_{k}^{\prime}$. Therefore now we can choose the $\varepsilon$ in (5.5) so small that, for each $f \in \mathcal{C}_{0, q}^{0}(X, V \otimes$ $E)$,

$$
\left\|\Phi_{j}^{-1} P\left(\Phi_{j}-\Phi_{k}\right) f\right\| \leq \frac{1}{2}\|f\| \quad \text { on } \quad \bar{U}_{j} \cap \bar{U}_{k}, \quad 1 \leq j, k \leq N .
$$

Take $\mathcal{C}^{\infty}$ functions $\chi_{j}: X \rightarrow[0,1], j=1, \ldots, N$, such that the family $\chi_{1}^{2}, \ldots, \chi_{N}^{2}$ forms a partition of unity subordinated to the covering $U_{1}, \ldots, U_{N}$. Set $\lambda_{j}=\chi_{j}^{2}$. Define bounded linear operators

$$
T_{r}^{\prime}, T_{r}^{\prime \prime}: \mathcal{C}_{0, r}^{0}(X, V \otimes E) \rightarrow \mathcal{C}_{0, r-1}^{\alpha}(X, V \otimes E), \quad r=q, q+1
$$

(where $T_{0}^{\prime}:=T_{0}^{\prime \prime}:=0$ if $q=0$ and $T_{n+1}^{\prime}:=T_{n+1}^{\prime \prime}:=0$ if $q=n$ ) and

$$
K^{\prime}, L^{\prime}, S, K^{\prime \prime}, L^{\prime \prime}: \mathcal{C}_{0, q}^{0}(X, V \otimes E) \rightarrow \mathcal{C}_{0, q}^{\alpha}(X, V \otimes E)
$$


setting

$$
\begin{aligned}
& T_{r}^{\prime} f=\sum_{j, k=1}^{N} \lambda_{j} \Phi_{j}^{-1} A_{r} P \Phi_{k} \lambda_{k} f, r=q, q+1, \quad S f=\sum_{j, k=1}^{N} \lambda_{j} \Phi_{j}^{-1} P\left(\Phi_{k}-\Phi_{j}\right) \lambda_{k} f, \\
& K^{\prime} f=\sum_{j, k=1}^{N} \bar{\partial} \lambda_{j} \wedge \Phi_{j}^{-1} A_{q} P \Phi_{k} \lambda_{k} f, \quad L^{\prime} f=-\sum_{j, k=1}^{N} \lambda_{j} \Phi_{j}^{-1} A_{q+1} P \Phi_{k}\left(\bar{\partial} \lambda_{k} \wedge f\right), \\
& T_{r}^{\prime \prime} f=\sum_{j=1}^{N} \chi_{j} \Phi_{j}^{-1} A_{r} Q \Phi_{j} \chi_{j} f, r=q, q+1, \\
& K^{\prime \prime} f=\sum_{j=1}^{N} \bar{\partial} \chi_{j} \wedge \Phi_{j}^{-1} A_{q} Q \Phi_{j} \chi_{j} f, \quad L^{\prime \prime} f=-\sum_{j=1}^{N} \chi_{j} \Phi_{j}^{-1} A_{q+1} Q \Phi_{j}\left(\bar{\partial} \chi_{j} \wedge f\right),
\end{aligned}
$$

where the notation is simplified: for example, $\Phi_{k}$ stands for id ${ }_{\Lambda^{0, q} T_{X}^{*}} \otimes \Phi_{k}$. Then it follows from Proposition 3.2, formula (5.3) and $\sum \lambda_{k} \equiv 1$ that

$$
\bar{\partial} T_{q}^{\prime} f+T_{q+1}^{\prime} \bar{\partial} f=\left(K^{\prime}+L^{\prime}+S\right) f+\sum_{j=1}^{N} \lambda_{j} \Phi_{j}^{-1} P \Phi_{j} f
$$

and

$$
\bar{\partial} T_{q}^{\prime \prime} f+T_{q+1}^{\prime \prime} \bar{\partial} f=\left(K^{\prime \prime}+L^{\prime \prime}\right) f+\sum_{j, k=1}^{N} \lambda_{j} \Phi_{j}^{-1} Q \Phi_{j} f .
$$

for all $f \in \widetilde{\mathcal{C}}_{0, q}^{0}(X, V \otimes E)$. Hence, setting $T_{r}:=T_{r}^{\prime}+T_{r}^{\prime \prime}$, we get

$$
\bar{\partial} T_{q}+T_{q+1} \bar{\partial}=\mathrm{id}+S+K^{\prime}+L^{\prime}+K^{\prime \prime}+L^{\prime \prime} \quad \text { on } \quad \widetilde{\mathcal{C}}_{0, q}^{0}(X, V \otimes E) .
$$

Lemma 5.1. The operators id $+t S, 0 \leq t \leq 1$, are isomorphisms of $\mathcal{C}_{0, q}^{0}(X, V \otimes$ $E)$, and $K^{\prime}, L^{\prime}, K^{\prime \prime}, L^{\prime \prime}$ are compact as endomorphisms of $\mathcal{C}_{0, q}^{0}(X, V \otimes E)$.

Proof. If we introduce in $\mathcal{C}_{0, q}^{0}(X, V \otimes E)$ the max-norm with respect to the fiber norm of $V \otimes E$ chosen above, then, by (5.7), the corresponding operator norm of $t S$ is $\leq t / 2$. Hence id $+t S$ is invertible. The compactness of $K^{\prime \prime}$ follows, because the section $\operatorname{id}_{\Lambda^{0, q} T_{X}^{*}} \otimes Q$ of $\Lambda^{0, q} T_{X}^{*} \otimes V \otimes B$ is fiber compact, $A_{q}$ acts continuously from $\mathcal{C}_{0, q}^{0}(X, V \otimes B)$ to $\mathcal{C}_{0, q-1}^{\alpha}(X, V \otimes B)$ and therefore, by Proposition 2.8, $A_{q} Q$ is compact as operator acting between $\mathcal{C}_{0, q}^{0}(X, V \otimes B)$ and $\mathcal{C}_{0, q-1}^{0}(X, V \otimes B)$. The compactness of $L^{\prime \prime}$ follows in the same way. As $\sum \lambda_{v} \equiv 1, \sum \bar{\partial} \lambda_{j} \equiv 0$ and, by (5.6), $\Phi_{j}^{-1}=\Phi_{v}^{-1}-\Phi_{j}^{-1} \Gamma_{j v}$ on $U_{v} \cap U_{j}$, we see that

$$
K^{\prime} f=-\sum_{\nu, j, k=1}^{N} \lambda_{v} \bar{\partial} \lambda_{j} \wedge \Phi_{j}^{-1} \Gamma_{j \nu} A_{q} P \Phi_{k} \lambda_{k} f
$$


By means of Lemma 2.5 this shows that $K^{\prime}$ is compact, because $A_{q}$ acts continuously from $\mathcal{C}_{0, q}^{0}(X, V \otimes B)$ to $\mathcal{C}_{p, q-1}^{\alpha}(X, V \otimes B)$ and each $\Gamma_{j v}$ is fiber compact. Finally, as $\sum \lambda_{v} \equiv 1, \sum \bar{\partial} \lambda_{k} \equiv 0$ and, by (5.6), $\Phi_{k}=\Phi_{v}-\Gamma_{v k} \Phi_{k}$ on $U_{k} \cap U_{v}$, we see that

$$
L^{\prime} f=\sum_{\nu, j, k=1}^{N} \lambda_{j} \Phi_{j}^{-1} A_{q+1} P \Gamma_{\nu k} \Phi_{k}\left(\lambda_{\nu} \bar{\partial} \lambda_{k} \wedge f\right),
$$

which implies the compactness of each $L^{\prime}$ by means of Proposition 2.8 .

Now we set

$$
M(t)=\mathrm{id}+t\left(S+K^{\prime}+L^{\prime}+K^{\prime \prime}+L^{\prime \prime}\right) \quad \text { for } \quad 0 \leq t \leq 1 .
$$

By Lemma 5.1, each $M(t)$ is a Fredholm operator in $\mathcal{C}_{0, q}^{0}(X, V \otimes E)$. It follows from (5.8) that $\mathcal{Z}_{0, q}^{0}(X, V \otimes E)$ is an invariant subspace of $M(1)$. This implies that $\mathcal{Z}_{0, q}^{0}(X, V \otimes E)$ is also an invariant subspace of $S+K^{\prime}+L^{\prime}+K^{\prime \prime}+L^{\prime \prime}=M(1)-\mathrm{id}$ and, hence, each of the operators $M(t)$, i.e.

$$
N(t):=\left.M(t)\right|_{\mathcal{Z}_{0, q}^{0}(X, V \otimes E)}
$$

is an endomorphism of $\mathcal{Z}_{0, q}^{0}(X, V \otimes E)$ for all $t \in[0,1]$. Since $M(t)$ is Fredholm, the kernel of $N(t)$ is of finite dimension and the image of $N(t)$ is topologically closed. Since $N(t)$ depends continuously on $t$, this implies that either, for all $t \in$ $[0,1]$, the image of $N(t)$ has infinite codimension in $\mathcal{Z}_{0, q}^{0}(X, V \otimes E)$, or, for all $t \in$ $[0,1]$, the image of $N(t)$ has finite codimension in $\mathcal{Z}_{0, q}^{0}(X, V \otimes E)$. As $N(0)=$ id, we conclude that the image of $N(t)$ has finite codimension for all $t \in[0,1]$. In particular, the space

$$
\left(\mathrm{id}+S+K^{\prime}+L^{\prime}+K^{\prime \prime}+L^{\prime \prime}\right)\left(\mathcal{Z}_{0, q}^{0}(X, V \otimes E)\right)
$$

is of finite codimension in $\mathcal{Z}_{0, q}^{0}(X, V \otimes E)$. As, by (5.8), this space is zero if $q=0$ and contained in $\bar{\partial} \widetilde{\mathcal{C}}_{0, q-1}^{0}(X, V \otimes E)$ if $q \geq 1$, this implies (5.1).

\section{References}

[1] L. Bungart, On analytic fiber bundles - I. Holomorphic fiber bundles with infinite dimensional fibers, Topology 7 (1968), 55-68.

[2] K. Clancey and I. Gohberg, "Factorization of Matrix Functions and Singular Integral Operators", OT 3, Birkhäuser, 1981.

[3] M. ERAT, The cohomology of Banach space bundles over 1-convex manifolds is not always Hausdorff, Math. Nachr. 248-249 (2003), 97-101.

[4] I. GOHBERG, The factorization problem for operator functions (Russian), Izv. Akad. Nauk SSSR Ser. Mat. 28 (1964), 1055-1082. 
[5] I. GOHBERG and J. LEITERER, The local principle for the factorization problem of continuous operator functions (Russian), Rev. Roumaine Math. Pures. Appl. 18 (1973), 1585-1600.

[6] G. Henkin and J. LeitereR, "Theory of Functions on Complex Manifolds", AkademieVerlag Berlin, 1984, and Birkhäuser, 1984.

[7] G. Henkin and J. LeITERER, "Andreotti-Grauert Theory by Integral Formulas", Progress in Mathematics, Vol. 74, Birkhäuser, 1988.

[8] J. LeITERER, From local to global homotopy formulas for $\bar{\partial}$ and $\bar{\partial}_{b}$, In: "Geometric Complex Analysis", J. Noguchi et al. (eds.), World Scientific Publishing Co., 1996, 385-391.

[9] J. Lindenstrauss and L. TZAFRIRI, "Classical Banach Spaces” I and II, Springer, 1996.

Institut für Mathematik

Humboldt-Universität zu Berlin

Rudower Chaussee 25

D-12489 Berlin, Germany

leiterer@mathematik.hu-berlin.de 